\title{
IDEAL FRAME AND MULTI-REVOLUTION METHODS FOR SPACE DEBRIS DYNAMICS
}

\author{
C. CALVO, B. MELENDO AND M. PALACIOS \\ Grupo de Mecánica Espacial, Universidad de Zaragoza \\ Zaragoza, Spain
}

\begin{abstract}
Using the computational advantages offered by the formulation of orbital problems with respect to the ideal frame, with or without regularization, we consider the multi-revolution methods to obtain numerically the short and long-term propagation of the resulting debris clouds after a breakup event of an operational satellite. The problem posed and solved in this form gives high efficiency. We think that software with these features will serve two purposes: it will support studies of the hazards of space debris for operational orbital space systems and also will help to create debris analysis programs more transferable and reliable.
\end{abstract}

\section{Introduction}

Several approximations have been developed around the analysis of the debris problem, undoubtedly due to the daily growing quantity of uncontrolled artificial material orbiting near the Earth.

Serious efforts have been made to incorporate in one package all the aspects relative to the simulation of break-up events and the posterior propagation of the debris particles after explosion or collisions. A sample of this effort is the DAW (Debris Analysis Workstation) (Bellman, 1993) software containing several moduli like: IMPACT, for the study of the break-up of objects upon impact and the formation of debris clouds; DEBRIS, for short-term debris propagation; LIFETIME, FOOTPRINT, EVOLVE, for long-term debris propagation.

The authors (Landauer, 1993; Bellman, 1993) still proclaim that a way of computing a better model for debris cloud expansion is needed, as well as improving the forecast of the debris environment and describing the potential threat of orbital debris to operational satellites in their lifetime (a few years). For that, short-term and long-term propagation of the debris

I. M. Wytrzyszczak, J. H. Lieske and R. A. Feldman (eds.), Dynamics and Astrometry of Natural and Artificial Celestial Bodies, 375, 1997.

(C) 1997 Kluwer Academic Publishers. Printed in the Netherlands. 
clouds, with the same values of the parameters and different random number seeds, will be necessary. As a consequence, more efficient numerical algorithms must be developed.

In the last few years, we have done research in the formulation of models which render very intuitive the dynamical evolution of the orbital parameters. Simultaneously, the equations can be integrated numerically with smallest loss of accuracy and great efficiency (Palacios and Calvo, 1996). We have developed the software called 'INPAID' (Calvo, Melendo and Palacios, 1995) which includes several models of formulation of orbital problems and several numerical step-by-step propagators [namely, $R K(8)$ and $\left.\mathrm{P}(\mathrm{EC})^{2} \mathrm{E}\right]$. More recently, we have incorporated an updated version of the multi-revolution methods, that we have formalized theoretically (Melendo and Palacios, 1996), with the possibility of step-size and order control.

In this paper, we present concisely the content of 'INPAID' software together with the multi-revolution methods. After a break-up event of an operational satellite simulated by ourselves, we make application to the short and long-term propagation of debris particles. The results are shown by a graphical display, created by our group, enabling us to visualize the dynamical evolution of the debris.

\section{INPAID: A Solver for Short-Term Propagation}

INPAID is a FORTRAN software package that we have implemented to numerically propagate orbital problems established as a first order initial value problem:

$$
\left\{\begin{array}{l}
y^{\prime}(t)=f(t, y(t)), \quad t \in\left[t_{0}, L\right], \quad y \in \mathbf{R}^{m} \\
y\left(t_{0}\right)=y_{0}
\end{array}\right.
$$

The solution is obtained in a grid of points defined as

$$
\left\{t_{j}=t_{0}+j h \mid j=0, \ldots, N=\left(L-t_{0}\right) / h\right\} .
$$

Three models (Palacios and Calvo, 1996) for the equations of motion have been considered, dubbed 'cd', 'piq' and 'pirq', respectively. The model 'cd' describes the motion in Cartesian coordinates with respect to the departure frame, an orthogonal reference defined by the initial position and velocity vectors. The model 'piq' uses an eighth order differential system (more equations than degrees of freedom, with constraints) to define the motion in projective coordinates with respect to the ideal frame using quaternions. The ideal frame is defined by means of a small rotation of the departure frame around the position vector, which transforms the $O x y$ plane onto the instantaneous orbital plane. In the model 'pirq', a regularization and a new independent variable, like the true anomaly, are additionaly included. 
In the last two models, the motion is decomposed into two non-independent parts: the motion of the ideal frame (defined by their quaternion) and the instantaneous motion of the particle on the orbital (ideal) plane.

Two numerical propagators have been included: a) a Runge-Kutta of order 8 with 13 stages and constant step-size; b) a predictor-corrector in the $P(E C)^{m} E$ mode, with $\mathrm{k}$ stages $(\mathrm{k}=6,8,10)$ and order $\mathrm{k}+2$. The predictor is an explicit Adams-Bashforth and the corrector is an optimal AS:S with stability properties, order $k+2$.

A simple analysis of the final global error gives the results below.
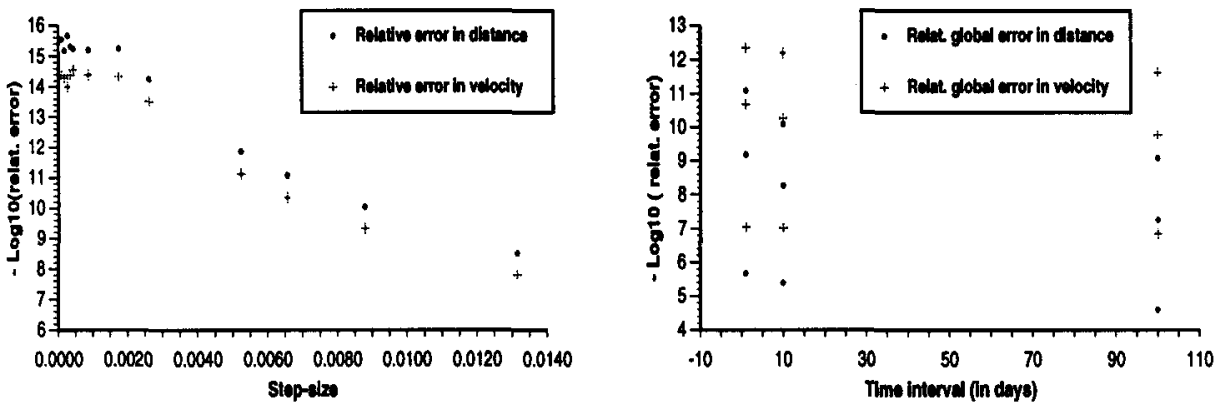

Figure 1. The final global error after 5 minutes and several step-sizes (left), and after 1,10 and 100 days and several step-sizes (right).

In Figure 1 (left), the relative global error in distance and velocity, when compared with a reference orbit, has been displayed for several step-sizes $(1,2,3,4,5,10,20,30,60,75,100$ and 150 seconds, which corresponds to $h \in[0.0001,0.0014]$, approximately), after 5 minutes, for a low orbit of initial semi-major axis, $a=6942.147 \mathrm{~km}, e=0.1$, perturbed by the Earth potential (zonal and tesseral harmonics). Both propagators, RK and $P(E C)^{2} E$, give almost the same figures.

In Figure 1 (right), the relative global error in distance and velocity has been displayed for an orbit of initial semi-major axis, $a=30466.024 \mathrm{~km}$, $e=0.431441$, perturbed by the zonal and tesseral harmonics up to order 6 . This global error has been calculated by forward and backward integration after 1,10 and 100 days, taking step-size of integration 10 minutes, 16 minutes and 32 minutes. For the last case, the measured CPU time was $3.312 \mathrm{~s}, 33.284 \mathrm{~s}$, and $333.318 \mathrm{~s}$, respectively.

Although the results presented here have been obtained with the model 'piq', this behavior is quite similar for the other models ('cd' and 'pirq') and numerical integrators, although the 'pirq' model shows slightly better results for eccentric orbits because of the regularization.

From these experiments we deduce that, when a step-by-step numerical method is used, to reach more than a few days within the required accuracy 
is very expensive, if not impossible, because the accumulated truncation error and excessive CPU are time consuming (small efficiency).

Thus, the idea to consider numerical methods enabling the orbit propagation during long periods of time and maintaining the same accuracy as the classical RK or multi-step methods with small cost is welcome.

\section{Improving the Performance of INPAID with Multi-Revolution Methods}

In contrast to step-by-step propagators, multi-revolution methods provide the solution to the difference equation

$$
y_{n+1}-y_{n}=g_{n}, \quad n \in N
$$

corresponding to a quasi-periodic motion, where $y_{n}$ is the approximate solution of (1), in a grid

$$
\left\{\bar{t}_{j}=t_{0}+j H \mid j \geq 0, H=M T, \text { and } M \text { a fixed integer }\right\}
$$

by means of the following multi-step-like algorithm:

$$
\sum_{j=0}^{\bar{k}} \bar{\alpha}_{j}(1 / M) \bar{y}_{n+j}=M \sum_{j=0}^{\vec{k}} \bar{\beta}_{j}(1 / M) \bar{g}_{n+j}, M \geq 1, n \geq 0,
$$

Some theoretical aspects of these methods, such as construction, stability, convergence, error bounds, etc. have been studied (Melendo and Palacios, 1996). In particular, we have achieved the control of the error propagation, an essential part of the application.

\section{Breakup of Satellite}

Instead of relying on an existing good model of a break-up event, we have produced 'at home' break-up simulations enabling us, in easy and accessible way, to apply the described software to the propagation of the debris particles.

We impose conservation of mass and momentum and small variation of energy due to the explosion or collision according to the type of breakup event considered (see for instance, Bellman 1993); besides, we consider that each element of the fragmentation will move with a velocity comparable in norm to the velocity of the satellite before the break-up.

We generate several random samples with a small number of fragments, using the Bayes Principle of Indifference (Landauer, 1993; McKnight 1991) to fill in values for the velocities of the debris particles after the explosion or collision. 
For the graphical output we have applied the just mentioned method to a geostationary satellite break-up, because in that area there exists a lot of operational objects (Grigorief, Sochilina and Vershkov, 1993) and it is known (Utkin and Chekalin, 1993) that in the past few years some of them have exploded.

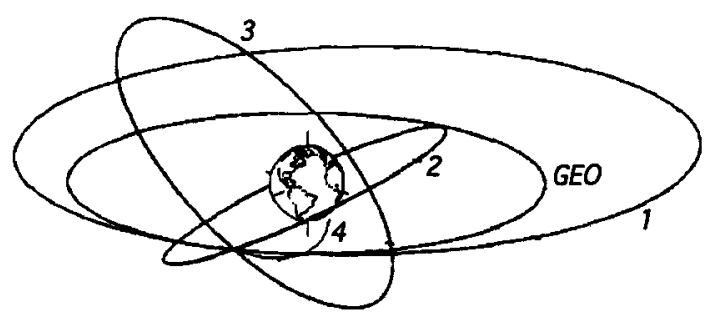

Figure 2. Short-term propagation. Evolution of the fragments after 16 days.

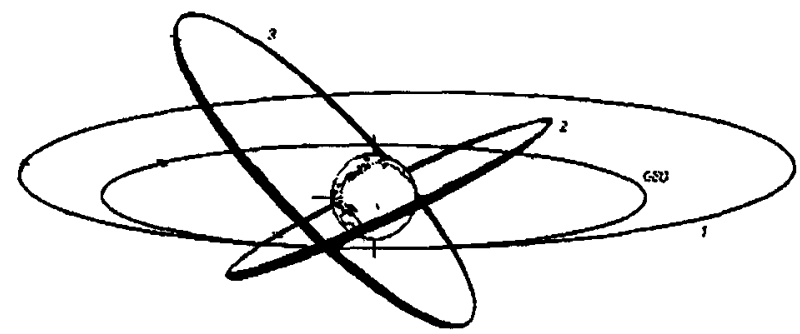

Figure 3. Long-term propagation. Evolution of the fragments after 160 days.

\section{Numerical Results and Graphical Output}

Trying to portray graphically the prediction of a long-term debris flux, we have made use of a software produced by the Grupo de Mecánica Espacial which takes advantage of the graphics facilities of the Silicon Graphics machine. The visual capabilities of that machine give the option of observing the particles moving along the orbit, previously computed. That gives the opportunity of following several of them and their possible encounters.

One version of this software displays on the screen a Mercator projection of the Earth and the orbits of the particles. A second version gives a 3-D representation of a rotating Earth together with the whole orbits fixed in the space frame and the particles moving each one on its orbit.

In Figure 2, we show one of these 'frozen' images corresponding to the orbits of the four particles originated after the simulated explosion of a 
METEOSAT satellite and the orbit of this satellite. The whole orbits have been propagated step-by-step during an interval of 16 days, taking into account the perturbation due to the Earth potential (only $J_{2}$ harmonic).

The eccentricity of the orbits span the range 0.0014 to 0.45 , and the period of the orbits are very different for each one. A judicious criterion should push to use the more adequate formulation of the dynamical model; this is possible in INPAID.

In Figure 3, we show another of these 'frozen' images corresponding to the same simulated explosion. In this case, the whole orbits have been propagated during an interval of 160 days taking into account the perturbation due to the Earth potential (only $J_{2}$ ).

\section{Conclusions}

INPAID package software, which is a good tool for short-term numerical integration of orbital problems, together with multi-revolution methods enables long-term propagation maintaining the accuracy. For this reason, we think that it can be a way of computing debris cloud expansion. The graphic package created in GME is a good tool to visualize debris motion. We are confident that this package will be a better way to support studies of hazards of space debris.

Acknowledgements. Partial support comes from the Spanish Ministry of Education CICYT \#PB94-5250).

\section{References}

Bellman, K.L.: 1993, "Debris analysis workstation: A modeling environment for studies on space debris", in: First European Conference on Space Debris (W. Flury, ed.), European Space Agency, Darmstadt, 303-308.

Calvo, C., Melendo, B., and Palacios, M.: 1995, "The choice of appropriate reference systems in numerical integration of orbits", Cahiers du Centre Européen de Géodynamique et de Séismologie 10, 99-108.

Grigorief, K.V., Sochilina, A.S and Vershkov, A.N.: 1993, "On catalogue of geostationary satellites", in: First European Conference on Space Debris (W. Flury, ed.), European Space Agency, Darmstadt, 665-669.

Landauer, C.: 1993, "Theoretical and practical issues in software for space debris modeling", in: First European Conference on Space Debris (W. Flury, ed.), European Space Agency, Darmstadt, 299-302.

McNight, D.: 1991, "Determination of breakup initial conditions", J. Spacecraft 28, 4, 470-477.

Melendo, B. and Palacios, M.: 1996, "A new approach to the construction of multirevolution methods and their implementation", Appl. Num. Maths. 20,1, 1-16.

Palacios, M. and Calvo, C.: 1996, "Ideal frame and regularization in numerical orbit computation", J. Astronaut. Sci. 44, 1, 63-77.

Utkin, V.F. and Chekalin, S.V.: 1993, "Space debris and orbital flight safety", in: First European Conference on Space Debris (W. Flury, ed.), European Space Agency, Darmstadt, 559-562. 